\title{
LA GUÍA DIDÁCTICA, UN MATERIAL EDUCATIVO PARA PROMOVER EL APRENDIZAJ E AUTÓNOMO. EVALUACIÓN Y MEJ ORAMIENTO DE SU CALIDAD EN LA MODALIDAD ABIERTA Y A DISTANCIA DE LA UTPL
}

\author{
(DIDACTIC GUIDE FOR PROMOTING SELF-STUDY THE. UTPL'S OPEN AND DISTANCE DE- \\ PARTMENT'S QUALITY ASSESSMENT AND IMPROVEMENT)
}

\author{
Ruth Marlene Aguilar Feijoo \\ Universidad Técnica Particular de Loja, UTPL (Ecuador)
}

\section{RESUMEN}

Uno de los cambios importantes que la Modalidad Abierta y a Distancia de la Universidad Técnica Particular de Loja introduce en su modelo pedagógico (1998-1999), se refiere a la sustitución de los textos (compilaciones) elaborados por los profesores de cada asignatura por libros convencionales, que si bien son actualizados, eligiendo lo mejor que existe en el mercado, a pesar de que no están diseñados para la enseñanza-aprendizaje a distancia. En estas circunstancias es cuando la Guía Didáctica se convierte en pieza clave para nuestro modelo, porque aproxima al alumno el material de estudio, potenciando sus bondades y compensando las limitaciones de los textos.

La Guía Didáctica es una herramienta valiosa que complementa y dinamiza el texto básico; con la utilización de creativas estrategias didácticas, simula y reemplaza la presencia del profesor y genera un ambiente de diálogo, para ofrecer al estudiante diversas posibilidades que mejoren la comprensión y el autoaprendizaje.

\begin{abstract}
One of the important changes that the UTPL's Open and Distance System pedagogical model (1998-1999) introduces is that instead of the texts (compilations) done by the tutors of each subject, conventional books are chosen. However, even though they are updated and the best that can be found on the market, they are not designed for distance teaching and learning. This is why the Didactic Guide becomes a key component for our model, because it brings the study material close to students, making the best use of its positive attributes and overcoming its limitations.
\end{abstract}

The Didactic Guide is a valuable tool complementing and making the basic text more dynamic. It is done using creative didactic strategies that simulate the presence of the tutor and generate a dialogue in order to offer students different possibilities to improve their understanding and the self-study process. 


\section{DESARROLLO}

La importancia y reconocimiento internacional alcanzado en los últimos años por la educación a distancia, la han convertido en la mejor alternativa para responder a los retos de formación y profesionalización permanente, a lo que se suma la exigencia que de manera creciente realiza la sociedad a las instituciones educativas, motivándolas a una constante evaluación y mejora de métodos, técnicas y materiales educativos, para llegar con una respuesta educativa de calidad.

En la búsqueda permanente de la calidad de los materiales educativos, en la Modalidad Abierta de la Universidad Técnica Particular de Loja (UTPL), se han dado los siguientes pasos (1998-2005):

1. Se abandona el sistema de enseñanza por objetivos.

2. Se reduce el número de evaluaciones a distancia de cuatro a dos, asignándoles un menor peso en la calificación total del 50 al 30\%.

3. Se empieza a utilizar textos convencionales o de mercado en lugar de las recopilaciones (antologías), elaboradas por los profesores.

4. Se crea y organiza el IPED.

5. Se introduce un nuevo concepto de Guía Didáctica; el de material educativo que orienta, motiva y conduce al estudiante paso a paso en el aprendizaje autónomo.

6. Se diseña un cuestionario completo de evaluación, que permite detectar las limitaciones en las Guías Didácticas.

7. Se inicia un proceso de evaluación periódica (semestral) de las Guías Didácticas, clasificándolas en A, B y C.

8. Se establece un programa de capacitación permanente a los profesores de las diferentes Unidades Académicas.

9. Se informa a las Unidades Académicas de las deficiencias que se observa en las guías y se realiza el seguimiento en el proceso de mejora.

10. Como experiencia piloto, se desarrolla un programa de tutoría y acompañamiento a los profesores de Ciencias Jurídicas en el mejoramiento de las de Guías Didácticas.

Comprometidos con la calidad y después de una experiencia en el diseño de materiales impresos, no muy positiva, se optó, en nuestro modelo educativo, por el uso de textos convencionales, que si bien son actualizados y lo mejor que existe en el mercado, no están diseñados con metodología para la enseñanza-aprendizaje a dis- 
tancia; es entonces cuando la Guía Didáctica cobra vital importancia, convirtiéndose en pieza clave, por las enormes posibilidades de motivación, orientación y acompañamiento que brinda a los alumnos, al aproximarles el material de estudio, facilitándoles la comprensión y el aprendizaje; lo que ayuda su permanencia en el sistema y suple en gran parte la ausencia del docente. De ahí la necesidad de que este material educativo esté didácticamente elaborado.

Es necesario puntualizar, que aunque la Modalidad Abierta y a Distancia de la UTPL cuenta con el apoyo de las nuevas TICs: un entorno virtual de aprendizaje (EVA), un sistema de Videoconferencia, que sin embargo aún no están al alcance de todos los alumnos, razón por la cual, la acción educativa continúa centrándose fundamentalmente en material impreso compuesto por:

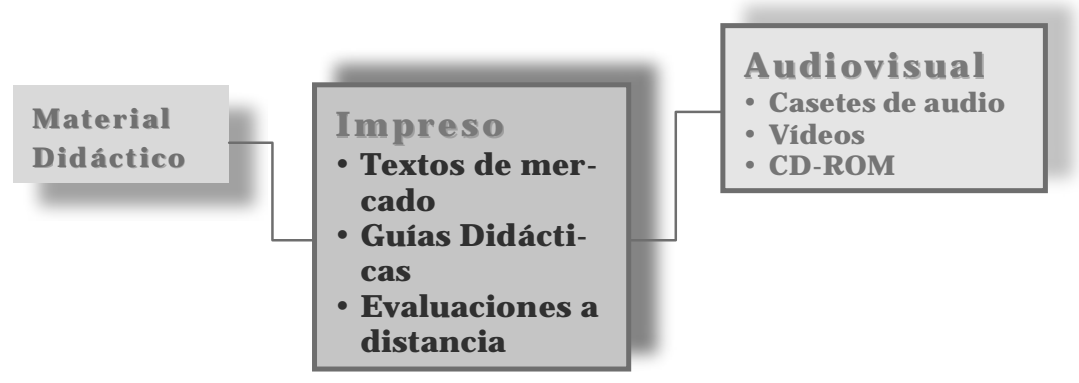

- Un texto básico convencional, seleccionado en función de criterios académicos de actualidad, rigor científico, calidad didáctica, congruencia con el programa de la asignatura y accesible en el mercado.

- Una Guía Didáctica, elaborada por el profesor de la asignatura, que motiva, orienta, promueve la interacción y conduce al estudiante, a través de diversos recursos y estrategias, hacia el aprendizaje autónomo.

- Las evaluaciones o trabajos a distancia, que posibilitan en los alumnos el desarrollo de diversas competencias: análisis, reflexión, aplicación de conocimientos, síntesis, evaluación, etc., a través de diversas tareas y ejercicios.

La Guía Didáctica es el tema que por ahora nos ocupa, por ello, empecemos observando con atención el siguiente gráfico, que pretende proporcionarnos una idea global de cómo surge y qué significa este material educativo. 


\section{¿Qué es una Guía Didáctica?}

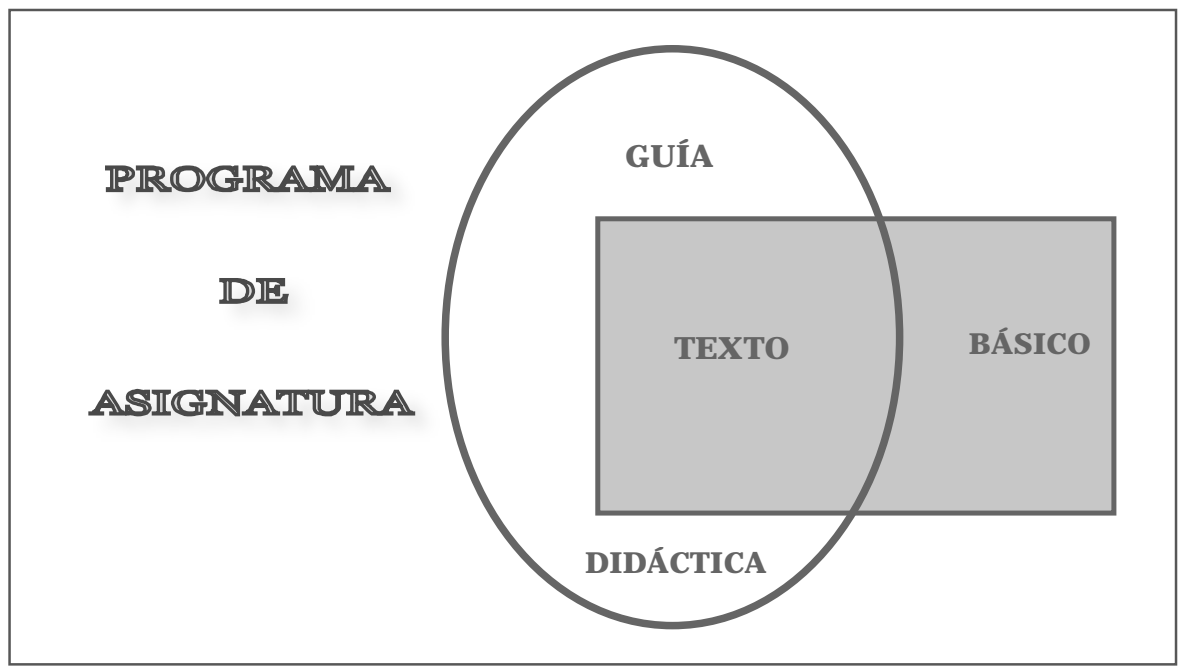

Después de observar el esquema precedente, intentaremos aproximarnos a una definición con la ayuda de expertos en este campo:

Para García Aretio (2002, p. 241) La Guía Didáctica es "el documento que orienta el estudio, acercando a los procesos cognitivos del alumno el material didáctico, con el fin de que pueda trabajarlos de manera autónoma".

Mercer, (1998: p. 195), la define como la "herramienta que sirve para edificar una relación entre el profesor y los alumnos".

Castillo (1999, p.90) complementa la definición anterior al afirmar que la Guía Didáctica es "una comunicación intencional del profesor con el alumno sobre los pormenores del estudio de la asignatura y del texto base [...]".

Para Martínez Mediano (1998, p.109) "constituye un instrumento fundamental para la organización del trabajo del alumno y su objetivo es recoger todas las orientaciones necesarias que le permitan al estudiante integrar los elementos didácticos para el estudio dela asignatura".

$\mathrm{Si}$ analizamos con detenimiento estas definiciones, con seguridad descubriremos aspectos muy importantes, que conviene destacar, para entender mejor el papel de la Guía Didáctica en la enseñanza-aprendizaje a distancia.

La definición primera nos habla de acercar el conocimiento al alumno; es decir, de allanar el camino para facilitar la comprensión de la asignatura; la segunda y tercera destacan la necesidad de la comunicación bidireccional o en palabras de 
Holmberg (1985) de "adoptar una actitud conversacional con el estudiante"; y la última rescata el papel orientador e integrador de la Guía Didáctica.

Personalmente considero que estos tres elementos que han sido contemplados en las definiciones anteriores constituyen los pilares sobre los que se construye y configura la calidad de las Guías Didácticas.

Esto nos permite sostener que la Guía Didáctica es el material educativo que deja de ser auxiliar, para convertirse en herramienta valiosa de motivación y apoyo; pieza clave para el desarrollo del proceso de enseñanza a distancia, porque promueve el aprendizaje autónomo al aproximar el material de estudio al alumno (texto convencional y otras fuentes de información), a través de diversos recursos didácticos (explicaciones, ejemplos, comentarios, esquemas y otras acciones similares a la que realiza el profesor en clase).

De ahí la necesidad de que la Guía Didáctica, impresa o en formato digital, se convierta en el "andamiaje" (J. Bruner) que posibilite al estudiante avanzar con mayor seguridad en el aprendizaje autónomo.

\section{¿POR QUÉ ES NECESARIO ELABORAR UNA GUÍA DIDÁCTICA?}

Con frecuencia los profesores se formulan esta pregunta. Las razones son varias. Intentemos señalar algunas:

- En la Modalidad a Distancia, debido a la separación física entre el profesor y los alumnos, no es posible una comunicación directa, cara a cara, entonces se tiene que recurrir a una comunicación mediada, que en nuestro país, básicamente se realiza a través de materiales impresos.

- En sistemas a distancia como el nuestro, que ha optado por textos convencionales o de mercado, que son libros eminentemente académicos y por lo mismo no están pensados para la enseñanza-aprendizaje a distancia, se hace indispensable elaborar Guías Didácticas que permitan "captar la atención del estudiante y compensar la presencia estimulante, motivadora y clarificadora del profesor de cada asignatura" (Marín Ibáñez, 1999,12).

- La modalidad a distancia plantea cambios en el papel del profesor, quien deja de ser el transmisor directo de los conocimientos para convertirse en el mediador, que orienta el trabajo independiente del alumno, que asume una función protagónica en el aprendizaje.

- La dificultad de conseguir en el mercado un texto que desarrolle íntegramente los contenidos del programa de la asignatura; de ahí la necesidad de organizarlos, profundizar o completar su desarrollo. 
- Los textos de mercado, por lo general, requieren adaptación al contexto en que se desarrolla la acción formativa ya sea en ejemplos o en datos estadísticos, etc.

- La necesidad de integrar en un solo documento las bondades de las guías de lectura, los cuadernillos de ejercicios y evaluación, y además, todas las orientaciones y estrategias que conduzcan al estudiante a abordar con éxito el aprendizaje autónomo.

\section{¿CUÁLES SON LAS FUNCIONES BÁSICAS DE LA GUÍA DIDÁCTICA?}

La Guía Didáctica cumple diversas funciones, que van desde sugerencias para abordar el texto básico, hasta acompañar al alumno a distancia en su estudio en soledad. Cuatro son los ámbitos en los que se podría agrupar las diferentes funciones:

a. Función motivadora:

- Despierta el interés por la asignatura y mantiene la atención durante el proceso de auto estudio.

- Motiva y acompaña al estudiante través de una "conversación didáctica guiada”. (Holmberg, 1985).

b. Función facilitadora de la comprensión y activadora del aprendizaje:

- Propone metas claras que orientan el estudio de los alumnos.

- Organiza y estructura la información del texto básico.

- Vincula el texto básico con los demás materiales educativos seleccionados para el desarrollo de la asignatura.

- Completa y profundiza la información del texto básico.

- Sugiere técnicas de trabajo intelectual que faciliten la comprensión del texto y contribuyan a un estudio eficaz (leer, subrayar, elaborar esquemas, desarrollar ejercicios...).

- "Suscita un diálogo interior mediante preguntas que obliguen a reconsiderar lo estudiado” (Marín Ibáñez, 1999).

- Sugiere distintas actividades y ejercicios, en un esfuerzo por atender los distintos estilos de aprendizaje.

- Aclara dudas que previsiblemente pudieran obstaculizar el progreso en el aprendizaje.

- "Incita a elaborar de un modo personal cuanto va aprendiendo, en un permanente ejercicio activo de aprendizaje" (Marín Ibáñez, 1999).

- Especifica estrategias de trabajo para que el alumno pueda realizar sus evaluaciones a distancia.

c. Función de orientación y diálogo:

- Fomenta la capacidad de organización y estudio sistemático.

- Promueve la interacción con los materiales y compañeros. 
- Anima a comunicarse con el profesor-tutor.

- Ofrece sugerencias oportunas para posibilitar el aprendizaje independiente.

d. Función evaluadora:

- Activa los conocimientos previos relevantes, para despertar el interés e implicar a los estudiantes. (Martínez Mediano, 1998: p.107)

- Propone ejercicios recomendados como un mecanismo de evaluación continua y formativa.

- Presenta ejercicios de autocomprobación del aprendizaje (autoevaluaciones), para que el alumno controle sus progresos, descubra vacíos posibles y se motive a superar las deficiencias mediante el estudio.

- Realimenta constantemente al alumno, a fin de provocar una reflexión sobre su propio aprendizaje.

- Especifica los trabajos de evaluación a distancia.

\section{ESTRUCTURA DE LA GUÍA DIDÁCTICA}

Cuando se ha elegido trabajar con textos convencionales o de mercado, como es nuestro caso, es indispensable elaborar Guías Didácticas muy completas, que potencien las bondades y compensen los vacíos del texto básico; para lo cual hemos optado por una Guía Didáctica que contemple los apartados siguientes:

1. Datos informativos.

2. Índice.

3. Introducción.

4. Objetivos generales.

5. Contenidos.

6. Bibliografía.

7. Orientaciones Generales.

8. Orientaciones específicas para el desarrollo de cada unidad.

- Unidad/número y título.

- Objetivos específicos.

- Sumario (temas de la unidad).

- Breve introducción.

- Estrategias de aprendizaje para conducir a la comprensión de los contenidos de la asignatura.

- Autoevaluación.

9. Soluciones a los ejercicios de autoevaluación.

10. Glosario.

11. Anexos.

12. Evaluaciones a distancia.

En esta propuesta de Guía Didáctica todos los elementos antes señalados son importantes y necesarios; pero existen dos en los que, de manera especial, se debe 
poner en juego la creatividad y la habilidad docente para conducir y generar aprendizajes; por lo tanto, es a los que nos referiremos en esta oportunidad.

\section{ORIENTACIONES GENERALES PARA EL ESTUDIO}

Constituyen sugerencias, consejos o ayudas que el profesor propone a los alumnos para que organicen mejor su tiempo, busquen condiciones favorables para estudiar, controlen sus progresos y obtengan satisfacción en sus estudios.

En nuestra Modalidad a Distancia, todos los alumnos reciben una Guía Didáctica específica para cada asignatura. Este material de apoyo se ha convertido en la herramienta principal de comunicación entre profesores y alumnos. Por eso quienes tienen la responsabilidad de su elaboración, no pueden conformarse con una simple presentación secuenciada de resúmenes; se requiere organizar y orientar el trabajo del alumno, anticipándose a las posibles dificultades que se pudieran presentar al abordar la información del texto básico o en el proceso mismo de aprendizaje.

\section{Algunas orientaciones generales que se recomienda incluir son:}

- Presentación del profesor o profesores que realizarán el seguimiento del proceso de enseñanza- aprendizaje.

- Descripción de los materiales y recursos con los que contará el alumno para el estudio de la asignatura.

- Horas requeridas para el estudio de la asignatura; se podría sugerir un calendario tentativo.

- Recomendaciones sobre algún método de estudio. Conviene orientar a los alumnos sobre la metodología propia de cada disciplina.

- Explicación de la importancia de la autoevaluación.

- Conveniencia de mantenerse en contacto con el profesor-tutor, ya sea a través del teléfono, correo electrónico o el entorno virtual de aprendizaje, para resolver dudas e inquietudes que pudieran surgir en el proceso de aprendizaje.

- Es muy importante recomendar algunas estrategias para abordar el texto de manera eficaz y evitar así la actitud frecuente de memorizar sin comprender.

El diagrama siguiente resume los aspectos fundamentales de las orientaciones generales de la Guía Didáctica. 


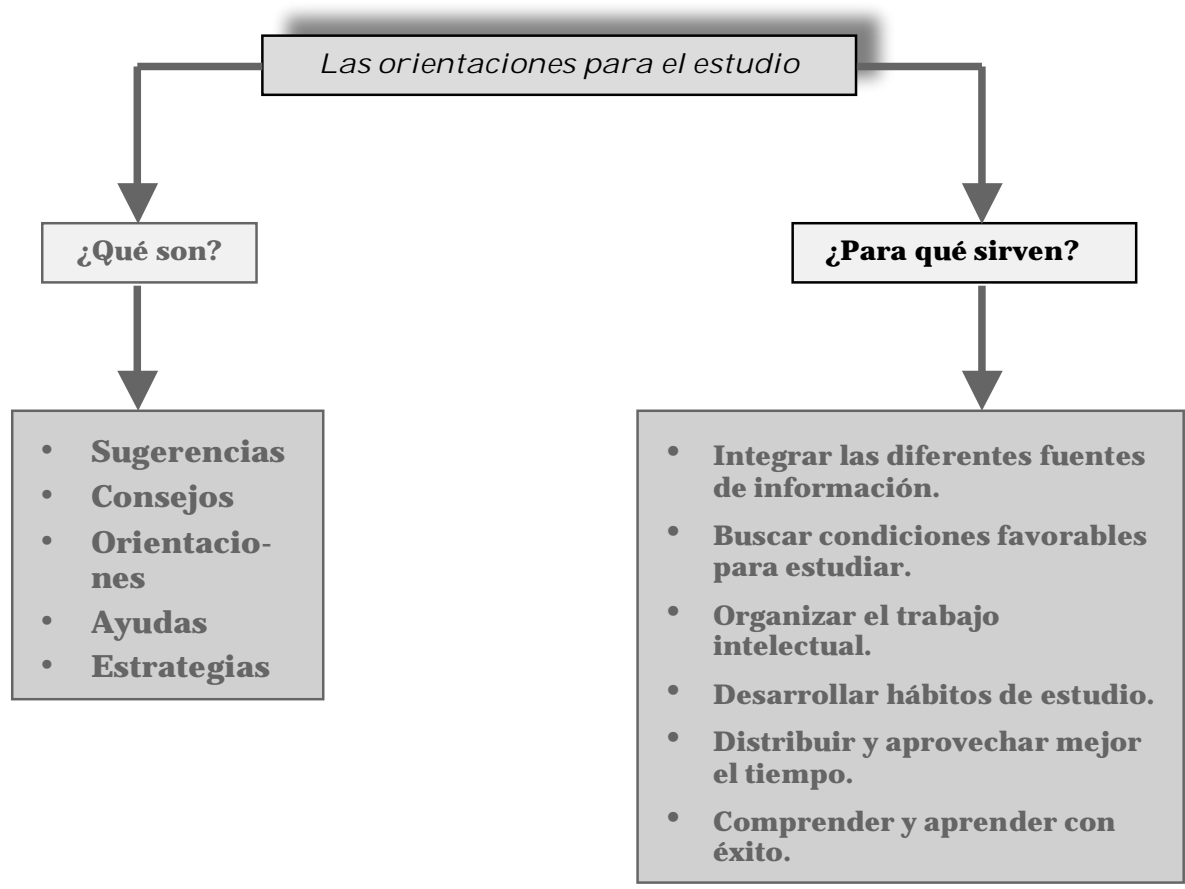

\section{DESARROLLO DEL APRENDIZAJE. ORIENTACIONES ESPECÍFICAS PARA EL ESTUDIO DE LAS UNIDADES Y TEMAS DE LA ASIGNATURA.}

Este es el apartado de mayor contenido, el elemento medular y divergente de la Guía Didáctica, a través del cual podemos valorar el ingenio, creatividad y capacidad docente de quienes diseñan este material escrito. Constituye el elemento central de la Guía Didáctica.

Para desarrollar esta parte el profesor necesitará recurrir a su experiencia docente, a su ingenio y creatividad para encontrar los recursos y estrategias didácticas que le posibiliten la comunicación con los alumnos y la consecución de los objetivos propuestos.

Aunque las Guías Didácticas tienen de manera general, una misma estructura, los recursos y estrategias de aprendizaje que se incluyen en cada una de ellas son específicos y estarán en función de la asignatura y de las bondades y limitaciones del texto seleccionado como básico; por lo tanto, antes de elaborar una Guía Didáctica conviene atender la sugerencia que hace el Instituto Universitario de Educación a Distancia (IUED) de la UNED, sobre la necesidad reflexionar en torno a las siguientes interrogantes (IUED, 1990, p9). 
a. ¿Qué tipo de texto básico se va utilizar?

b. ¿Cómo está estructurado?

c. ¿Qué ayudas incluye?

d. ¿Qué nivel de autosuficiencia tiene el texto?

De las respuestas que podamos dar, dependerá el tipo y variedad de ayudas que se requerirá incorporar en las Guías Didácticas, a fin de enriquecer y complementar los textos convencionales, con la metodología de enseñanza a distancia.

Existe un conjunto amplio de recursos y estrategias para orientar el desarrollo de cada uno de los temas, acercar la información al alumno y facilitar la comprensión. A continuación señalamos algunos, pero será cada profesor quien con el dominio del contenido científico y sus habilidades didácticas, seleccione los recursos que mejor respondan a las características de los destinatarios y a los contenidos que desea enseñar.

Las estrategias y ayudas para facilitar la comprensión textual, pueden dirigirse básicamente a dos ámbitos (Solé, 2002, 34 y 38).

A. Intervenir sobre el texto básico: ya sea para explicar, completar, ejemplificar, esquematizar, profundizar o resumir la información que contiene en sus páginas.

B. O en relación al sujeto que aprende, motivándole a través de actividades o ejercicios variados como: establecer el propósito de la lectura, activar los conocimientos previos, centrar la atención mediante preguntas intercalas, ayudar a discriminar la información importante de lo accesorio, fomentar la reflexión y el análisis, para que el alumno "no se limite a memorizar y aplique constantemente los conocimientos convirtiéndolos en algo operativo y dinámico" (Loockwood, citado por García Aretio, 2002, p. 237), lo que indudablemente favorecerá la comprensión.

Hemos encontrado diversas clasificaciones de los recursos, ayudas o estrategias de aprendizaje, pero la clasificación que nos ofrece el IUED (1990, 10-11) nos parece práctica y por lo mismo la hemos tomado para nuestro trabajo. Esta clasificación, realizada en función de la oportunidad con que se ofrecen las ayudas a los alumnos, considera tres momentos:

1. Estrategias previas al estudio: el docente puede acudir a diversas ayudas o recursos didácticos que le permitan contextualizar e introducir al estudiante en cada unidad o bloque temático. Algunos recursos que se podrían utilizar son:

- Objetivos específicos que focalizan la atención del estudiante respecto a los logros que se esperan de él con el estudio de la unidad.

- Sumario, o esquema de contenidos, que contribuye a establecer la macroestructura del texto. 
- Introducción, o planteamiento breve de la unidad, con la finalidad de crear expectativas y despertar el interés por el tema.

- Conocimientos previos: conviene especificar las habilidades y conocimientos requeridos para el estudio de la asignatura o módulo, así por ejemplo, se podría incluir un cuestionario sobre los conocimientos básicos que el estudiante debe saber antes de abordar el nuevo tema o sugerir algún texto que le ayude a recordar esos elementos necesarios.

- Orientación bibliográfica de la unidad: se podría especificar el capítulo o capítulos que se trabajará en la unidad o sugerir otra bibliografía de ampliación del tema.

2. Estrategias paralelas al estudio: dependiendo de las bondades del texto, el docente necesitará apoyarse en diferentes recursos didácticos para conducir al estudiante en su proceso de aprendizaje autónomo. Entre las ayudas paralelas que se podría utilizar están:

- Preguntas intercaladas que dirigen la atención hacia los aspectos esenciales del tema.

- Ejercicios que permitan relacionar los conocimientos que posee el alumno con la nueva información.

- Organizadores previos (Ausubel): un esquema, un mapa conceptual o un diagrama...

- Ejemplos, analogías, metáforas...

- Gráficos, fotografías, tablas...

- Anticipar dificultades frecuentes con las que suelen encontrarse los alumnos.

- Completar y profundizar algunos temas.

- Desarrollar temas que no constan en el texto.

- Guías de lectura para conducir al alumno a través del texto básico.

- Ofrecer resúmenes, esquemas de los temas, especialmente de los más complejos.

- Sugerir el desarrollo de ejercicios propuestos en el texto básico (si los hay).

- Comentarios sobre cómo abordar ciertos temas complejos o puntos obscuros.

Pero no todo tiene que hacerlo el profesor, conviene mantener un sano equilibrio, recordando siempre, Lo que pueda hacer el alumno, no lo haga el profesor (Valero, 1975). Es bueno facilitarle los recursos, abrir caminos y ofrecerle posibilidades, orientándole a realizarlas; sin dejar de solicitar a los alumnos la elaboración de esquemas, resúmenes, gráficos, ejemplos, pequeñas investigaciones de campo, visitas guiadas y otros ejercicios de refuerzo y aplicación de los conocimientos.

\section{Estrategias posteriores al estudio}

Dirigidas al autocontrol de los progresos en el aprendizaje y a la preparación para la evaluación presencial, entre las que podemos destacar: 
- Auto evaluaciones.

- Distintas actividades sugeridas como por ejemplo, consultas en otras fuentes.

- Desarrollo de las evaluaciones a distancia.

Finalmente, a manera de conclusiones podemos señalar:

1. La actualización y mejoramiento de la calidad pedagógica de los materiales educativos que se utilizan en educación a distancia es una tarea permanente.

2. En la Modalidad a Distancia de la UTPL, en el proceso de mejora de los materiales impresos, distinguimos tres momentos bien diferenciados:

a. El primero con una duración de 12 años (1976-1990) en el que se manejaban textos de mercado y los profesores elaboraban las "Programaciones" (especie de cuadernillo de evaluaciones a distancia); material que contenía: los objetivos, esquema de contenidos y bibliografía de la asignatura; además, las evaluaciones a distancia con sus correspondientes estrategias de trabajo.

b. El segundo que va desde 1991 a 1998 (8 años) y que se denominó modelo de “aprendizaje por dominio y evaluación criterial". Período en el que los profesores elaboraban los textos específicos para cada asignatura y, una Guía Didáctica breve que incluía: objetivos generales, contenidos, bibliografía, un plan de evaluación muy detallado con objetivos operativos y algunos ejercicios de evaluación formativa (diagnóstica y autoevaluaciones).

c. La última etapa, en la que nos encontramos actualmente, se inicia en 1999 comprende cambios fundamentales como: se abandona la enseñanza por objetivos, se seleccionan los mejores textos que existen en el mercado y se elaboran guías mucho más completas y didácticas, que cumplan realmente las funciones de motivar, orientar, facilitar el aprendizaje y evaluar.

3. Se inicia un proceso de evaluación periódica (semestralmente) de todas las Guías Didácticas, clasificándolas en tres categorías, debiendo mejorarse inmediatamente las que se ubicaban en la categoría "C".

4. Se realizan jornadas permanentes de formación y capacitación del personal docente, de las diversas Unidades Académicas, para el diseño y mejoramiento de este material educativo.

5. En los últimos tres años se advierte una mejora substancial en la calidad de las Guías Didácticas tanto de fondo como en la forma: estructura interna, externa y presentación. 


\section{REFERENCIAS BIBLIOGRÁFICAS}

Álvarez, M.A. (1997): Destrezas de la Lectura, en torno a la metodología de ense ñanza a distancia, Madrid, Ed. UNED.

Bruer, J.T. (1999): Escuelas para pensar, una ciencia de aprendizaje en el aula, Madrid, Ediciones Paidós.

Corral iñigo, A., Tejero Escribano, L. y Otros. (1987): Consideraciones acerca de la realización de textos didácticos para la enseñanza a distancia, Madrid, UNED.

Díaz Barriga, A. y otros (1988) Estrategias de aprendizaje para la comprensión de textos académicos en prosa. En: Perfiles Educativos, núm. 41-42.

García Aretio, L. (2002): La Educación a Distancia, dela teoría a la práctica, Madrid, Ed. Ariel, S.A.

- (Coord.) (1997): Aprender a distancia, Estudiar en la UNED, Madrid, Ed. UNED.

- (Coord.) (1997): Unidades didácticas y Guías Didácticas, orientaciones para su elaboración, Madrid, Ed. UNED.

- (Ed.) (1998): El material impreso en la enseñanza a distancia, Madrid, Ed. UNED.

Gutiérrez Martínez, F. Los procesos de comprensión de textos [en línea]. Disponible en: http://virtual3.uned,es/web-medioct/courses/870802 5/tesinas/L02cle/F Gutiérrez.htm [consulta 2004, 21 de abril].

Instituto Universitario de Educación a Distancia, IUED (1990): Elaboración de Guías Didácticas. Consideraciones y Orientaciones, p. 9.

Marín Ibáñez, R. (1999): El Aprendizaje abierto y a distancia, el material impreso, Loja-Ecuador, Ed. UTPL.

Santamaría De reyes, P. (1989): Estrategias metodológicas par ala producción de material didáctico en la educación a distancia, Revista Iberoamericana de Educación Superior a Distancia, Vol. 1 No. 3, Madrid, Ed. UNED.

Solé, I. (2002): Estrategias de lectura, Barcelona-España, Ed. Graó.

Unía, M.E. (2001): Estrategias Didácticoorganizativas para mejorar los centros educativos, Madrid, Narcea S.A. Ediciones.

Valero, J .M. (1975): Educación Personalizada, ¿Utopía o Realidad?, p.

\section{PALABRAS CLAVE}

Guía Didáctica, modelo pedagógico, interacción, comprensión, textos convencionales, estrategias didácticas, evaluaciones a distancia.

\section{KEY WORDS}

Didactic Guide, pedagogical model, interaction, comprehension, conventional texts, didactic strategies, distance education. 


\title{
PERFIL ACADÉMICO DE LA AUTORA
}

Ruth Marlene Aguilar Feijoo, Dra. en Psicología Educativa por la Universidad Nacional de Loja y Máster en EaD por la UNED. Es profesora de la Universidad Técnica Particular de Loja y Coordinadora del Instituto de Pedagogía para la Educación a Distancia (IPED).

Dirección postal:

\author{
Ruth Marlene Aguilar Feijoo \\ Universidad Técnica Particular de Loja \\ Loja - Ecuador \\ E-mail: raguilar@utpl.edu.ec
}

Fecha recepción del artículo: 20. 03. 2006

Fecha aceptación del artículo: 31. 03. 2006 